\title{
Profiling bacterial community in upper respiratory tracts
}

Hana Yi ${ }^{1,2,3}$, Dongeun Yong ${ }^{4}$, Kyungwon Lee ${ }^{4}$, Yong-Joon Cho ${ }^{5}$ and Jongsik Chun ${ }^{5,6^{*}}$

\begin{abstract}
Background: Infection by pathogenic viruses results in rapid epithelial damage and significantly impacts on the condition of the upper respiratory tract, thus the effects of viral infection may induce changes in microbiota. Thus, we aimed to define the healthy microbiota and the viral pathogen-affected microbiota in the upper respiratory tract. In addition, any association between the type of viral agent and the resultant microbiota profile was assessed.

Methods: We analyzed the upper respiratory tract bacterial content of 57 healthy asymptomatic people (17 health-care workers and 40 community people) and 59 patients acutely infected with influenza, parainfluenza, rhino, respiratory syncytial, corona, adeno, or metapneumo viruses using culture-independent pyrosequencing.

Results: The healthy subjects harbored primarily Streptococcus, whereas the patients showed an enrichment of Haemophilus or Moraxella. Quantifying the similarities between bacterial populations by using Fast UniFrac analysis indicated that bacterial profiles were apparently divisible into 6 oropharyngeal types in the tested subjects. The oropharyngeal types were not associated with the type of viruses, but were rather linked to the age of the subjects. Moraxella nonliquefaciens exhibited unprecedentedly high abundance in young subjects aged $<6$ years. The genome of M. nonliquefaciens was found to encode various proteins that may play roles in pathogenesis.
\end{abstract}

Conclusions: This study identified 6 oropharyngeal microbiome types. No virus-specific bacterial profile was discovered, but comparative analysis of healthy adults and patients identified a bacterium specific to young patients, M. nonliquefaciens.

Keywords: Microbiome, Respiratory tract, Moraxella, Influenza, Oropharynx, Healthcare staff

\section{Background}

Recent culture-independent community analysis performed on the human microbiome has provided an overall picture of commensal microbial communities. Studies have revealed that diverse microbes occupying body habitats with strong niche specialization both within and among individuals [1]. In the case of the respiratory tract microbiome, a catalogue was initially established in 2009 [2] and then respiratory microorganisms were extensively characterized [3-8]. Collectively, studies to date have revealed that the respiratory tract harbors a homogenous microbiota that decreases in biomass from the upper to the lower tract [5], and that the lung microbiome resembles the oral microbiome,

\footnotetext{
* Correspondence: jchun@snu.ac.kr

${ }^{5}$ ChunLab, Inc., Seoul, Republic of Korea

${ }^{6}$ School of Biological Sciences \& Institute of Bioinformatics (BIOMAX), Seoul National University, Seoul, Republic of Korea

Full list of author information is available at the end of the article
}

although these microbiomes are distinguished by the overrepresentation of distinct bacterial species in the lung [7]. As with other human-body habitats, the core microbiome of nasopharynx remains undefined because it varies substantially from person to person [3]. However, existence of core microbiome was observed despite the significant inter-individual variation [8]. One study reported that the microbial composition of the upper respiratory tract is typically unique to each person and it changes little over time [4].

Although the available evidence is not sufficiently strong, microbiome types are speculated to eventually affect a person's risk of disease or response to distinct drugs [9]. The human microbiota is considered to benefit the host by promoting the differentiation of the mucosal structure and function, stimulating both the innate and adaptive immune systems, and providing "colonization resistance" against pathogen invasion [10]. Recently, the 
composition of the airway microbiota has been suggested to play roles in determining the presence and severity of diseases [11,12]. For example, the clinical outcomes of respiratory infections caused by Pseudomonas aeruginosa vary depending on the diversity of the airway microbiota $[13,14]$, and a temporal loss of the diversity is linked to the development of ventilator-associated pneumonia and patient mortality $[12,13]$. The importance of intact commensal microbiota was also demonstrated in viral infection, with the commensal microbiota composition critically regulating host immune response following influenza virus infection [15]. To reveal the links that exist between microbiome types and clinical traits, we have to first understand the diversity of the microbial community in target body sites.

Most respiratory tract infections are caused by viruses including rhinovirus, respiratory syncytial virus, parainfluenza virus, adenovirus, coronavirus, human metapneumovirus, and influenza virus. Infection by pathogenic viruses significantly changes the condition of the respiratory tract as a result of the epithelial damage caused by viral invasion itself and/or by inflammatory mediators produced by the host immune response [16]. Given, the pathophysiology and mechanism of local immune responses are virus specific [16], a virus-specific bacterial profile in the respiratory tract could potentially be characterized. Discovering any specific bacterial species that exhibits a tendency of opportunistic infection or coinfection in a viral species-dependent would benefit future preventive measures and current treatments. To date, no study has evaluated whether the composition of the respiratory microbiota changes in relation to the type of infectious virus.

In this study, our aim was to determine whether a viral infection-related bacterial profile exists in the respiratory tract and evaluate any disparities in the microbiota structure that develops depending on the infectious virus species. We used culture-independent high-throughput sequencing to analyze the bacterial content in the upper respiratory tract of patients and healthy asymptomatic people. We also examined the presence or absence of dissimilarities in the microbiota of hospital staff and community people.

\section{Methods}

\section{Ethics statement}

This study was approved by the Institutional Review Board of the Severance Hospital, Yonsei University Health System, Seoul, Korea (protocols 4-2010-0652, 4-2011-0159, and 4-2011-0862). Patients and healthy adults provided written informed consent to be enrolled. De-identified demographic data and clinical measures were taken from electronic medical record system. Additional file 1: Table S1 presents the list and features of samples used in this study.

\section{Subjects and sample collection}

We selected 59 patients with confirmed acute viral infections from Yonsei University Hospital during a 30month period (December 2010 to May 2013). The viral agents of the infections were confirmed using PCR by Yonsei University Hospital. The viruses included influenza (IF, $\mathrm{n}=7$ ), parainfluenza (PI, $\mathrm{n}=24)$, rhino $(\mathrm{RH}$, $\mathrm{n}=8)$, respiratory syncytial $(\mathrm{RS}, \mathrm{n}=14)$, corona $(C R$, $\mathrm{n}=4)$, adeno $(\mathrm{AD}, \mathrm{n}=1)$, and metapneumo $(\mathrm{MP}, \mathrm{n}=1)$ viruses. The upper respiratory tract samples were collected from patients' oropharynx by using swabs and suspended in $1 \mathrm{~mL}$ of viral transport medium (VTM; Becton Dickinson Universal Viral Transport, USA). Sputum or nasopharyngeal aspirate was collected when available instead of swabs. Sputum samples were diluted with an equal volume of suspension medium and homogenized as described [17]. The upper respiratory tract samples were also obtained from healthy adults including 17 health-care workers (9 non-ICU and 8 ICU staff) and 40 community people. The 17 hospital staffs and 7 community people were recruited over the same period in Yonsei University Hospital (June 2011) and 33 community people were additionally recruited in the same hospital (June 2013). The oropharyngeal swabs were obtained using aseptic technique, suspended in VTM and transported to the laboratory for further processing. The samples were stored at $-80^{\circ} \mathrm{C}$ until DNA extraction.

\section{DNA extraction, PCR, and pyrosequencing}

DNA was extracted from $200 \mu \mathrm{L}$ of samples by using a commercial microbial DNA isolation kit (Qiagen). The extracted DNA was amplified using primers targeting the V1 to V3 regions of the prokaryotic 16S rRNA gene by using methods described elsewhere [18]. DNA was sequenced by Chunlab Inc. (Seoul, Korea) by using a Roche/454 GS Junior system according to the manufacturer's instructions. The processing of pyrosequencing data of $16 \mathrm{~S}$ rRNA gene sequences were performed as described elsewhere [18]. Chimeric sequences were detected using UCHIME [19] and EzTaxon-e database (http://eztaxon-e.ezbiocloud.net; [20]) was used to taxonomically assign each pyrosequencing read.

\section{Phylogenetic analyses}

Phylogenetic analyses of 16S rRNA gene sequences were performed using the neighbor-joining [21] tree method implemented in MEGA program [22]. An evolutionary distance matrix was generated for the neighbor-joining tree according to the model of Jukes and Cantor [23] and the resultant tree topologies were evaluated using bootstrap analyses [24]. 


\section{Genome analyses}

The draft genome sequence of Moraxella nonliquefaciens DSM $6327^{\mathrm{T}}$ was determined through paired-end shotgun sequencing performed by using the MiSeq system (Illumina) with $300 \times$ coverage. The sequencing reads were assembled using CLC genomics wb5 (CLCbio). Annotation, comparative genomic analyses and average nucleotide identity (ANI) calculation were performed as described [25].

\section{Statistical analyses}

Random subsampling was conducted to normalize the data size to 3,000 reads, because the total number of reads that remained after pre-processing varied depending on the samples. All statistical analyses were performed using this subset. The Simpson diversity index [26] was calculated using the rRNA Database Project's pyrosequencing pipeline (http://pyro.cme.msu.edu/). The overall phylogenetic distance between each pair of communities was estimated using the Fast UniFrac web interface (http://unifrac.colorado.edu/) [27] and visualized using principal coordinate analysis (PCoA) implemented in $\mathrm{R}$ program (http://www.r-project.org/). To compare microbiome structures based on categorical metadata, samples were pooled into binds (healthy/patient, male/female, VTM/aspirate/sputum, smoking/non-smoking, ages, causal viruses, etc.) and statistical significance tests were performed using $\mathrm{R}$ program. The significance of differences in bacterial profiles according to categorical metadata was determined using Hotelling's $t$ test. Significant bacterial taxa based on categorical metadata were identified using $q$-values after multiple testing correction [28] to eliminate false discovery rates. The difference in Shannon diversity index among categorical metadata was evaluated using Wilcox two-sample $t$ test.

\section{Availability of supporting data}

The 454 sequencing data supporting this article are available in the GenBank repository, SUB435282. The genome data of $M$. nonliquefacience is under submission to the DDBJ/EMBL/GenBank databases under accession No. PRJNA232737.

\section{Results}

\section{Sequencing statistics}

We sequenced 57 upper respiratory tract samples from healthy adults including 17 health-care workers (9 nonICU and 8 ICU staff) and 40 community people. The 59 patients with confirmed acute viral infections with influenza (IF, $\mathrm{n}=7$ ), parainfluenza $(\mathrm{PI}, \mathrm{n}=24)$, rhino $(\mathrm{RH}, \mathrm{n}=8)$, respiratory syncytial $(\mathrm{RS}, \mathrm{n}=14)$, corona $(\mathrm{CR}, \mathrm{n}=4)$, adeno ( $\mathrm{AD}, \mathrm{n}=1)$, and metapneumo (MP, $\mathrm{n}=1$ ) viruses were also successfully sequenced. The pyrosequencing of $16 \mathrm{~S}$ rRNA gene amplicons resulted in 786,152 quality-filtered reads for the 116 samples.
We observed an average of 823 bacterial phylotypes (97\% clustering) for each samples (range 237 to 1,851). In the sample-size-normalized (3,000 nt) subsamples, the number of bacterial species ranged from 180 to 615 (average, 371), depending on samples.

\section{Microbiota of healthy adults}

The genus Streptococcus was identified as the core microbiome of the healthy human respiratory tract. In all healthy subjects tested in this study, members of Streptococcus dominated the bacterial community, exhibiting an average abundance ratio (percentage of the taxon in the total bacterial community) of $55.8 \%$ (range $13.4 \%-91.1 \%$, depending on subjects) (Table 1). The genera Neisseria (8.0\%) and Gemella (5.3\%) were also dominant in healthy subjects, but their abundance ratios were considerably less than that of Streptococcus. The genera observed in all healthy subjects were Streptococcus, Prevotella, and Veillonella. The genera Haemophilus, Gemella, Rothia, and Leptotrichia were detected in most subjects at abundance ratios of $2.0 \%-8.0 \%$.

Fast UniFrac analyses for the bacterial profiles in healthy samples showed that hospital staff and community people were not discriminated based on their bacterial composition (Additional file 2: Figure S1), and age, sex, year and month of sample collection, and smoking status did not discriminate the bacterial profile (data not shown).

\section{Difference between patient and healthy-adult groups}

Analyzing the bacterial communities of healthy-adult and patient groups revealed clear differences. We used the Shannon index in which higher values represent higher diversities; the average values calculated for the healthy-adult and patient groups were $4.83 \pm 0.40$ and $3.77 \pm 0.61$, respectively (Figure 1 ). This indicated that healthy adults harbored significantly more complex and diverse microbiota than did patients $(p<0.0001$, Wilcox test).

The microbiota profiles of healthy-adult and patient groups also differed in the relative composition of the microbiome, which was highlighted in the graph showing the abundant bacterial genera observed in the tested samples (Figure 2 and Additional file 3: Figure S2). To identify the bacterial taxa that were more abundant (in a statistically significant manner) in the patient group than in the healthy-adult group, $p$-values were calculated for all the taxa detected. The result demonstrated that distinct bacterial genera were overrepresented in the patient and healthy-adult groups. Whereas Haemophilus $(\mathrm{p}=0.010)$ and Moraxella $(\mathrm{p}=0.028)$ were identified as patient group-specific genera, Streptococcus $(\mathrm{p}=0.003)$, Neisseria ( $\mathrm{p}=0.003)$, Gemella $(\mathrm{p}=0.003)$, Aggregatibacter $(\mathrm{p}=0.008)$, and Actinobacillus $(\mathrm{p}=0.001)$ were determined to be bacteria specific to the healthy-adult group. 
Table 1 List of dominant bacterial genera and their average abundance (\%) in samples

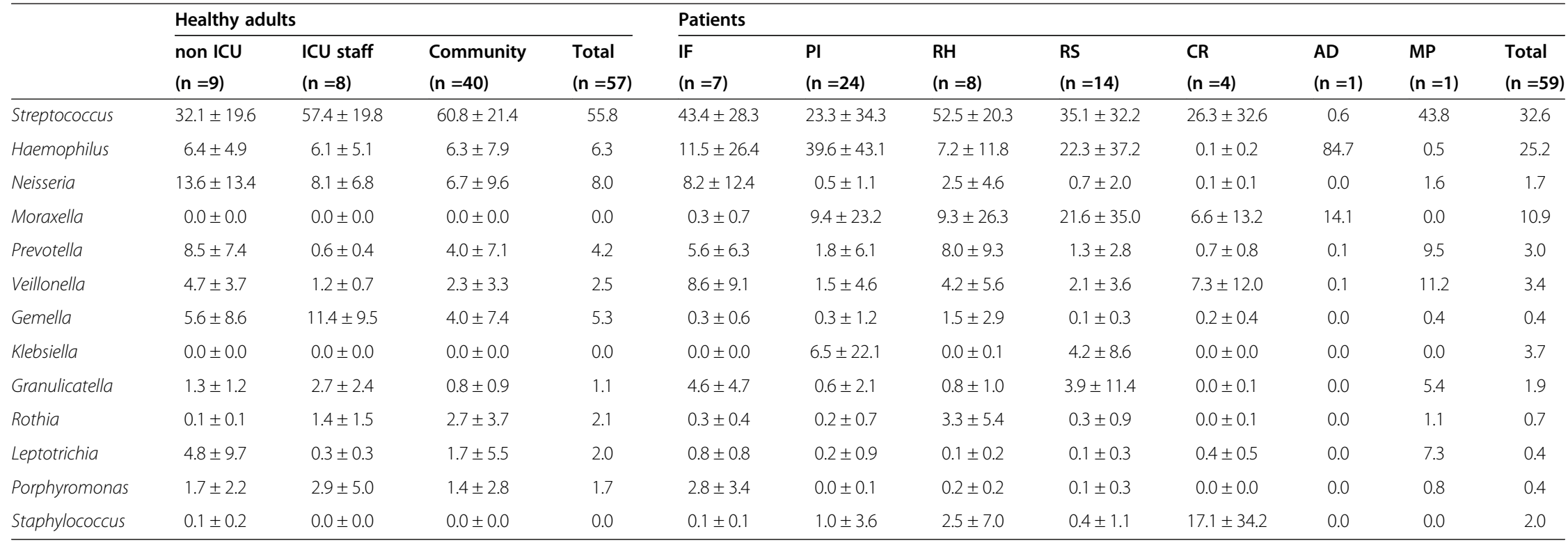

Abbreviations: ICE intensive care unit, non ICU healthy hospital staff who do not work in ICU, ICU staff healthy hospital staff who work in ICU, Community healthy community people, IF influenza, $P I$ parainfluenza, $R H$ rhino, $R S$ respiratory syncytial, $C R$ corona, $A D$ adeno $M P$ metapneumo. 


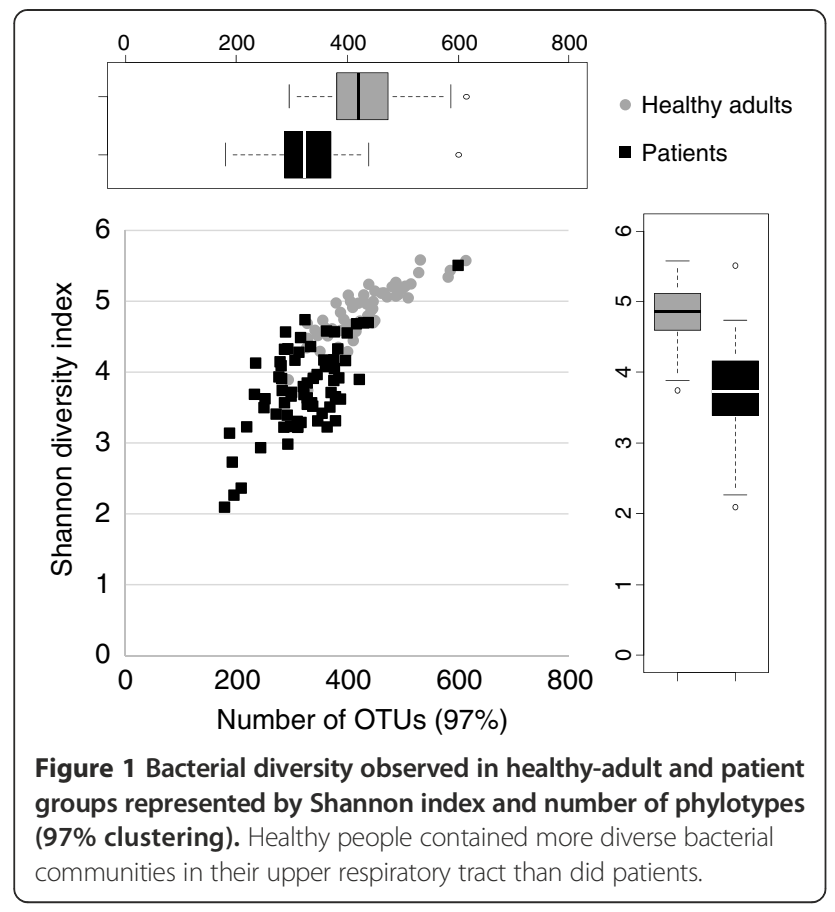

\section{Oropharyngeal microbiome types}

To hierarchically visualize the bacterial profile similarities among the samples, a UPGMA dendrogram was generated from the Fast UniFrac distance matrix. Based on bacterial composition, the samples analyzed in this study were divided into 6 oropharyngeal microbiome types (Additional file 3: Figure S2), with the clusters being characterized by the dominance of several bacterial genera: Type 1 (dominated by Streptococcus + Prevotella + Veillonella), Type 2 (Streptococcus + Haemophilus + Neisseria), Type 3 (Streptococcus), Type 4 (Moraxella), Type 5 (Haemophilus), and Type 6 (Klebsiella). Only 4 samples were not grouped into any of the 6 types. The healthy adults and a subset of patients harbored bacterial communities dominated by Streptococcus, and to a lesser extent by Haemophilus, Neisseria, Prevotella, Veillonella, and/or Gemella (Types 1-3 in Figure 3). The remaining patients carried impaired microbiota dominated by Moraxella (Type 4 in Figure 3), Haemophilus (Type 5), or Klebsiella (Type 6), and this was coupled with a massive reduction in the levels of Streptococcus. Types 5 and 6 were dominated by well-known pathogens like $H$. influenzae and $K$. pneumonia, but Type 4 was dominated by a previously unknown one, Moraxella nonliquefaciens.

\section{Effect of causal virus type and other variables on microbiota}

We elucidated the differences in bacterial profiles in the context of causal agents of infections and demonstrated that virus type did not determine the structural differences in bacterial communities (Additional file 4: Figure
S3A). Moreover, sex, sample type (swab, aspirate, or sputum), and smoking status did not influence the bacterial community structure (Additional file 4: Figures S3B, S3C and S3D), which was also unaffected by the year and month of sample collection (data not shown). By contrast, subjects' age was associated with the bacterial profile in a statistically significant manner (Additional file 4: Figure $\mathrm{S} 3 \mathrm{E}$ ), and the samples were categorized into 2 age groups, $0-5$ and $6-90$ years $(p<0.0001$, Hotelling's test).

\section{Moraxella nonliquefaciens and $M$. catarrhalis}

In this study, we discovered a bacterial species that was dominant in young patients ( $0-5$ years old): $M$. nonliquefaciens was detected in $32.2 \%$ of the patients, with abundance ratios of $0.03 \%-97.0 \%$ depending on the subject, but this species was not observed in any healthy subject. Most of the patients (16 out of 19 cases) harboring $M$. nonliquefaciens were under 6 years old. Two RSV infected patients (RS1 and RS7) showed 95 and 97\% of abundance of $M$. nonliquefaciens, indicating that the upper respiratory tracts of these patients were overwhelmed by this bacterial species. In addition, a closely related pathogenic species, $M$. catarrhalis, was detected in $23.7 \%$ of the patients, with abundance ratios of $0.03 \%-26.5 \%$.

Analyzing the $16 \mathrm{~S}$ rRNA gene sequence revealed that $M$. nonliquefaciens and $M$. catarrhalis were clearly distinct species that showed $98.4 \%$ similarity between type strains (Additional file 5: Figure S4). The Moraxella contigs recovered from patient samples were divided into 2 clades based on phylogenetic analysis (Figure 4). Clade I was closely related $(98.5 \%-99.8 \% 16 \mathrm{~S}$ rRNA gene similarity) to the type strain of $M$. nonliquefaciens. The branching pattern of the contigs within the radiation of Clade I indicated that $M$. nonliquefaciens populations in the patients encompassed 3 phylogenetic lineages. Contigs belonging to Clade II were clustered together with M. catarrhalis $(99.6 \%-100 \% 16$ S rRNA gene similarity), and these contigs were further divided into 2 subpopulations, Type 1 and Type 2 populations [29].

\section{Evaluating potential pathogenicity of $M$. nonliquefaciens based on genome sequences}

To determine whether $M$. nonliquefaciens has a possibility to be an opportunistic pathogen, its potential pathogenicity was inferred using the genome sequence of the type strain. Genome sequencing identified a 2.22-Mb-sized genome of $M$. nonliquefaciens DSM $6327^{\mathrm{T}}$, featuring a $42.06 \% \mathrm{G}+\mathrm{C}$ ratio. The genomic relatedness calculated using ANI showed that M. nonliquefaciens DSM $6327^{\mathrm{T}}$ and $M$. catarrhalis RH4 shared low genomic relatedness, $75 \%$ ANI, which is considerably less than the cut-off value used for species circumscription, 95\%-96\% ANI [30]. The ANI value further confirmed that the 2 species were distinct. 


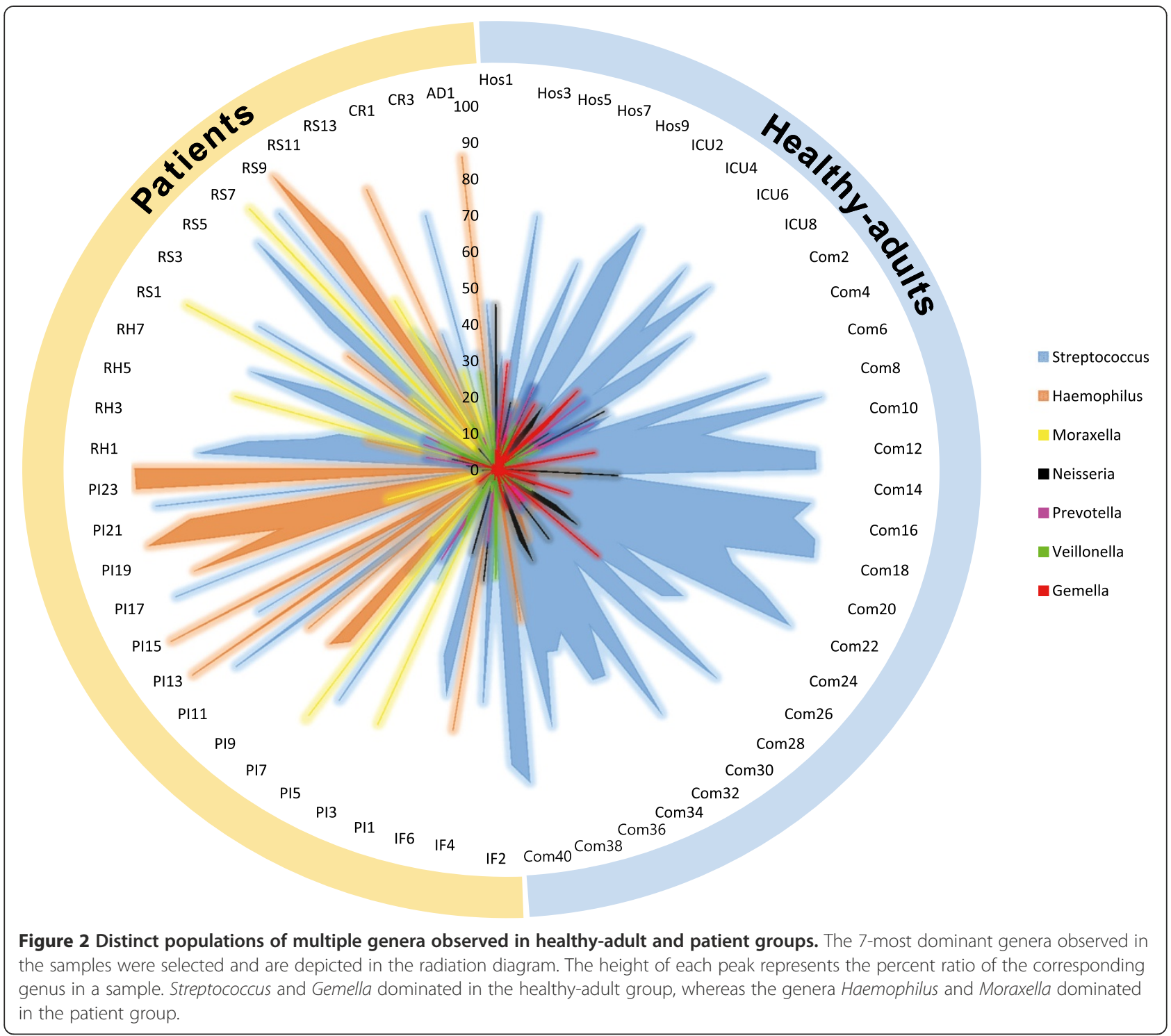

Various proteins have been reported to play pivotal roles in $M$. catarrhalis pathogenesis [31]. Thus, we examined whether the virulence proteins in the $M$. catarrhalis RH4 genome were also encoded in the newly determined $M$. nonliquefaciens genome. The genes responsible for host-cell adhesion and invasion, evasion of host immune system, and biofilm formation were included as putative virulence factors. Comparative genomic analysis revealed that most of the virulence genes identified in M. catarrhalis were encoded in the M. nonliquefaciens genome (Additional file 6: Table S2). Moreover, resistance to $\beta$-lactam antibiotics was predicted based on the presence of the $\beta$-lactamase Class $C$ gene. However, BRO-1 and BRO-2 $\beta$-lactamases encoded by most of $M$. catarrhalis strains were not detected in $M$. nonliquefaciens.

\section{Discussion}

Our results demonstrated that changes in bacterial profiles elicited by viral infection were not associated with the causal viral species: the microbiome compositions in samples obtained from various viral infections were not differentiated based on the causal infectious agents. Regardless of the causal agents involved, the respiratory tract microbiota of patients differed substantially from the microbiota of healthy subjects in the kinds and diversities of prevalent bacteria. However, the heterogenicity of the patient group of this study (age, sampling type, and number of samples/virus type) hinders strong conclusions for this point. Nevertheless, the current results from this study provide the first insight into microbiome alterations associated with viral infection in the upper respiratory tract. 


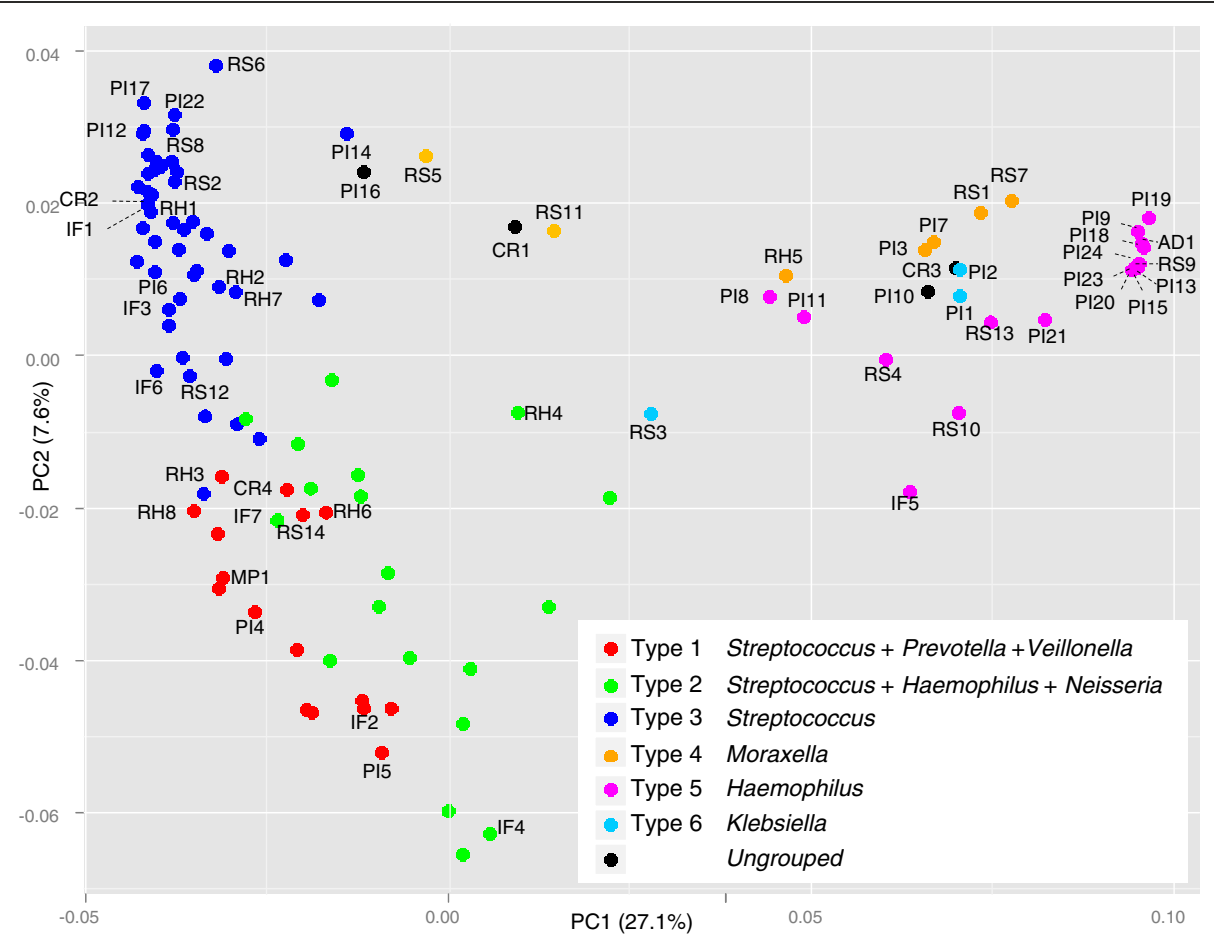

Figure 3 Dependence of microbiome structure on several key genera. Principal coordinate analysis (PCOA) of the bacterial communities isolated from 57 healthy-adult and 59 patient samples was performed using the weighted pairwise UniFrac distance matrix. The UniFrac distance represents the distance between 2 samples in terms of the microbial community structure. Samples are color-coded according to the 7 clustering groups.

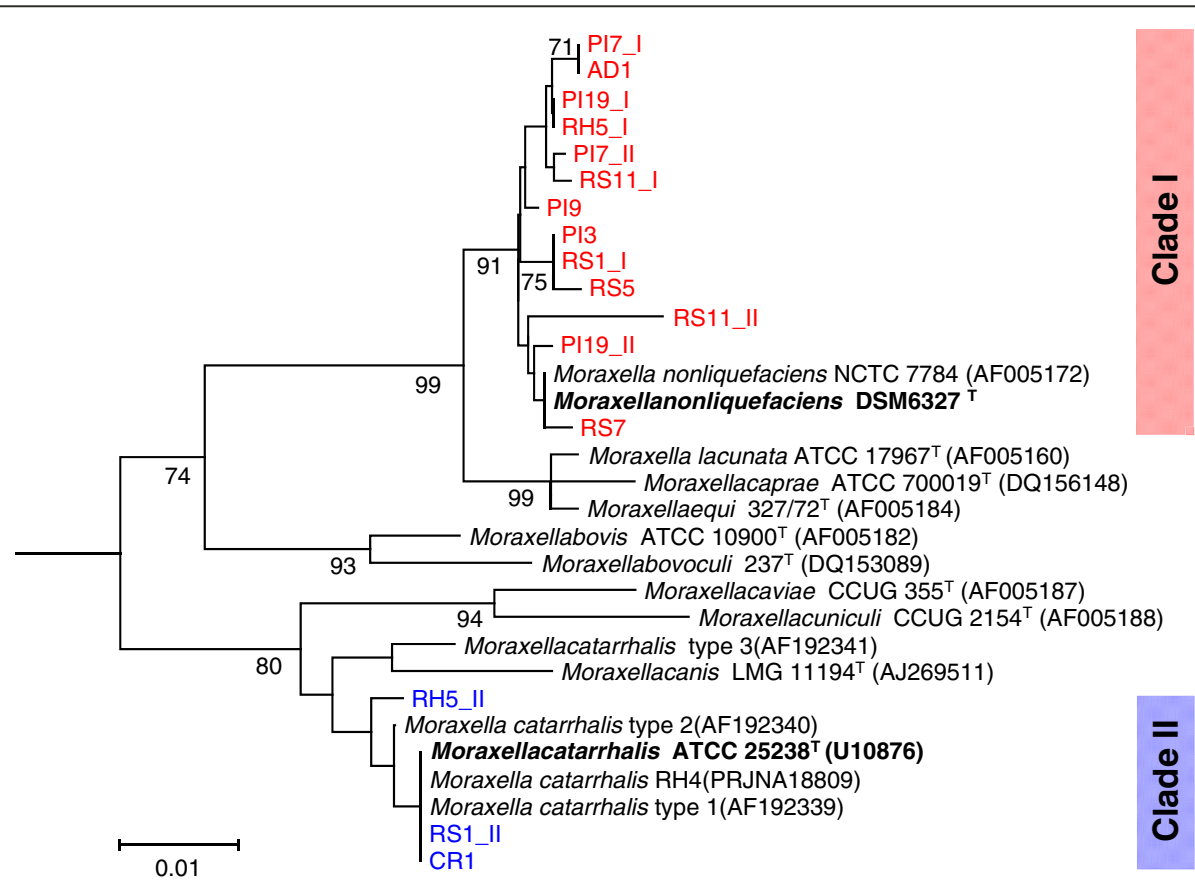

Figure 4 Phylogenetic tree based on 16S rRNA gene sequences showing the relationship of the newly discovered Moraxella sequences with publically available sequences of other Moraxella strains. Representatives of $3 \mathrm{M}$. catarrhalis type strains were included together with the type strains of Moraxella species. Red text and blue test indicate M. nonliquefaciens contigs and $M$. catarrhalis contigs recovered from patient samples, respectively. The neighbor-joining tree was evaluated using 1,000 bootstrap pseudoreplicates. Only bootstrap values over $70 \%$ are shown at branch nodes. The scale bar represents the genetic distance. 
The diminished bacterial diversity observed in patients agreed well with previous studies reporting that the diversity in commensal airway microbiota declined following infection by specific pathogens $[6,13]$. For example, in the nares of patients with $S$. aureus carriage, species diversity was half of that found in healthy adults' nares [6]. These results indicate that the normal microflora is depleted in respiratory tract cells impaired due to viral infection and is replaced by a few opportunistic pathogens. Moreover, the dominance of Streptococcus in the respiratory tracts of healthy subjects (Table 1) agreed with previous culture-independent massive metagenomic sequencing studies [3,7]. Streptococcus is also known to be abundant in the oral cavity $[32,33]$. Thus, the oropharynx of healthy people could be characterized by high bacterial diversity and by an overwhelming abundance of the genus Streptococcus.

By analyzing the bacterial community, we defined 6 oropharyngeal types of bacterial populations in the upper respiratory tract. We use the word "oropharyngeal type" here based on the "enterotype" concept, which was introduced by Arumugam et al. and defined as the clusters of human gut microbiome determined based on bacterial composition [34]. The concept suggests that people can be classified into several enterotypes according to the abundance of key bacterial taxa in gut microbial communities [35]. In this study, the samples included were restricted to one ethnic group, and thus the suggested 6 oropharyngeal types may be accepted only temporarily. However, because no other efforts to cluster respiratory tract microbiomes have been reported to date, our results may serve as a favorable starting point for future studies on this subject.

Our results revealed that Haemophilus and Moraxella were patient-specific genera. Unlike $H$. influenzae, $M$. nonliquefaciens has not been studied for its possible role in pneumonia. Although $M$. nonliquefaciens has been isolated from clinical cases including chronic bronchitis [36], bronchial infection [37], pneumonitis [38], endophthalmitis [39], septic arthritis [40], thyroiditis [41], discitis [42], botryomycosis [43], and endocarditis [44], this bacterium is widely considered to be a part of the normal flora in the human upper respiratory tract and to exhibit low pathogenicity $[45,46]$. By contrast, a closely related species, $M$. catarrhalis, is a well-studied respiratory tract pathogen that frequently colonizes the nasopharynx and is an exclusively human pathogen that displays an affinity for the human upper respiratory tract $[47,48]$. Long considered to be a commensal bacterium of the upper respiratory tract, $M$. catarrhalis has now been established as an etiological cause of otitis media and the exacerbations of chronic obstructive pulmonary disease (COPD) [47,49].

Despite the distinctiveness of the 2 species at the taxonomic level, $M$. nonliquefaciens and $M$. catarrhalis share several common features. First, the age-related incidence of $M$. nonliquefaciens infection determined here is concordant with that of $M$. catarrhalis. Previously, M. catarrhalis was reported to be mostly associated with upper respiratory tract infections in children [50], and its carriage rate was shown to be high in children (up to $75 \%$ ) and extremely low in healthy adults (1\%-3\%) [49,51-54]. Moreover, the phenotype and gene incidences of $M$. catarrhalis isolates of children and adults presenting with respiratory disease differ substantially, possibly as a result of immune evasion in adults [48]. The age-related incidence of $M$. nonliquefaciens and $M$. catarrhalis may be indicative of the weak pathogenicity of Moraxella species, which may be unable to evade the well-established immune system of adults.

Second, most strains of $M$. catarrhalis are known to produce $\beta$-lactamases and thus exhibit ampicillin resistance [49]. This antibiotic resistance was also predicted in $M$. nonliquefaciens based on the presence of the Class C $\beta$-lactamase gene. However, although both species possess Class $C \beta$-lactamase genes, the species differ with respect to the possession of $\mathrm{BRO} \beta$-lactamase; $\mathrm{BRO}$ is unique because it shows no substantial similarity to any $\beta$-lactamase genes identified so far [55]. The absence of BRO-1 and BRO-2 in M. nonliquefaciens suggests that $M$. catarrhalis acquired the BRO genes by means of lateral gene transfer after the 2 species evolved into distinct lineages.

Third, all but one virulence factors reported for $M$. catarrhalis were found to be encoded by $M$. nonliquefaciens (Additional file 6: Table S2), which indicates that $M$. nonliquefaciens has a high potential to be pathogenic even though it is currently considered to be a commensal bacterium. Several reasons may account for why the overabundance of $M$. nonliquefaciens has not been reported. Growing this organism and distinguishing it from $M$. catarrhalis are challenging, which may have resulted in a poor recognition of $M$. nonliquefaciens as a respiratory pathogen. Moreover, although $M$. catarrhalis is focused on by clinicians, the isolation of $M$. catarrhalis from clinical samples is complicated by the presence of Neisseria strains because these organisms share morphological similarities [49]. Furthermore, M. nonliquefaciens may have been considerably underestimated because of being misidentified as $M$. catarrhalis or Neisseria spp. [49]. Lastly, the current absence of clinical interest or familiarity with $M$. nonliquefaciens may have resulted in under-reporting or identification of this pathogen. Indeed, $M$. catarrhalis was previously underreported since other better recognized pathogens were also recognized and growing in the same cultures [49]. To clarify the incidence of this potential pathogen, future studies will need to differentiate between the true rates of incidence of $M$. catarrhalis and $M$. nonliquefaciens. The results of this study 
suggest that $M$. nonliquefaciens deserves considerable attention as a potential opportunistic pathogen in the respiratory tract.

\section{Conclusions}

The pyrosequencing of bacterial community identified 6 oropharyngeal microbiome types in the upper respiratory tract, but the bacterial profile was not related to the type of causal infected viruses. The microbiota of patients differed substantially from that of healthy subjects in the kinds and diversities of prevalent bacteria. Comparative analysis of healthy adults and patients identified a bacterium specific to young patients, $M$. nonliquefaciens. The results of whole-genome sequencing raised the possibility of $M$. nonliquefaciens being an opportunistic pathogen.

\section{Additional files}

Additional file 1: Table S1. List and clinical characteristics of samples used in this study. ND, not determined.

Additional file 2: Figure S1. Ordination diagram showing the relatedness of microbiomes in the upper respiratory tract of healthy people. Principal coordinate analysis (PCOA) of bacterial communities isolated from 57 healthy adults was performed using the weighted pairwise UniFrac distance matrix The UniFrac distance represents the distance between 2 samples in terms of the microbial community structure.

Additional file 3: Figure S2. Dendrogram and circle map showing the clustering of samples into 6 groups depending on bacterial population dynamics. The unweighted pair group method with arithmetic mean (UPGMA) dendrogram was generated from the Fast UniFrac distance matrix to hierarchically visualize the manner in which samples are grouped. The relative abundance of representative microbial genera is indicated as a circle map; circle sizes represent the percentage ratio within a sample.

Additional file 4: Figure S3. Ordination diagram showing the relatedness of microbiomes in the upper respiratory tract of people afflicted with diverse viral infections. We performed principal coordinate analysis (PCOA) on the bacterial communities isolated from 57 healthy-adult and 59 patient samples by using the weighted pairwise UniFrac distance matrix. The UniFrac distance represents the distance between 2 samples in terms of the microbial community structure. The structure of the bacterial community was not affected by (A) the type of virus or by (B) the sex, (C) sample type, and (D) smoking status of the subjects. However, (E) age was correlated with the microbiome structure.

Additional file 5: Figure S4. Diagram showing the relationships among the 2 type strains and the 2 representative contigs obtained from patient samples. The average nucleotide identity (ANI) value indicating genome relatedness was calculated using the complete genome sequence of $M$. catarrhalis RH4 strain (PRJNA 48809), which shows 100\% 165 rRNA gene sequence identity with the type strain of the species.

Additional file 6: Table S2. Comparison of major virulence genes present in M. nonliquefaciens and M. catarrhalis. The list of virulent genes was obtained from previous reports $[31,56]$. The gene locus in each genome is presented together with gene size in amino acids (in parenthesis).

\section{Abbreviations}

UPGMA: Unweighted pair group method with arithmetic mean PCoA: Principal coordinate analysis; ANI: Average nucleotide identity; VTM: Viral transport medium; ICU: Intensive care unit.

\section{Competing interests}

The authors declare that they have no competing interests.

\section{Authors' contributions}

HY, DY and JC conceived and designed research. DY and KL collected samples and clinical data. HY, YC and DY performed research. HY, DY, YC and JC analyzed data. HY, DY and JC wrote the paper. All authors read and approved the final manuscript.

\section{Acknowledgements}

We thank Dr. S Won for his advices on statistical analyses. This work was supported by research funds (2011-E43000-00, 2012-E43001-00, and 2013-E43001-00) of the Korea Centers for Disease Control and Prevention.

\section{Author details}

${ }^{1}$ School of Biosystem and Biomedial Science, Korea University, Seoul, Republic of Korea. ${ }^{2}$ Department of Public Health Science, Graduate School, Korea University, Seoul, Republic of Korea. ${ }^{3}$ Korea University Guro Hospital, Korea University, Seoul, Republic of Korea. ${ }^{4}$ Department of Laboratory Medicine and Research Institute of Bacterial Resistance, Yonsei University College of Medicine, Seoul, Republic of Korea. ${ }^{5}$ ChunLab, Inc., Seoul, Republic of Korea. ${ }^{6}$ School of Biological Sciences \& Institute of Bioinformatics (BIOMAX), Seoul National University, Seoul, Republic of Korea.

Received: 21 July 2014 Accepted: 23 October 2014

Published online: 13 November 2014

\section{References}

1. Human Microbiome Project C: Structure, function and diversity of the healthy human microbiome. Nature 2012, 486:207-214.

2. Costello EK, Lauber CL, Hamady M, Fierer N, Gordon Jl, Knight R: Bacterial community variation in human body habitats across space and time. Science 2009, 326:1694-1697.

3. Bogaert D, Keijser B, Huse S, Rossen J, Veenhoven R, Van Gils E, Bruin J, Montijn R, Bonten M, Sanders E: Variability and diversity of nasopharyngeal microbiota in children: a metagenomic analysis. PLoS One 2011, 6:e17035.

4. Charlson ES, Chen J, Custers-Allen R, Bittinger K, Li H, Sinha R, Hwang J, Bushman FD, Collman RG: Disordered microbial communities in the upper respiratory tract of cigarette smokers. PLoS One 2010, 5:e15216.

5. Charlson ES, Bittinger K, Haas AR, Fitzgerald AS, Frank I, Yadav A, Bushman FD, Collman RG: Topographical continuity of bacterial populations in the healthy human respiratory tract. Am J Respir Crit Care Med 2011 184:957-963.

6. Frank DN, Feazel LM, Bessesen MT, Price CS, Janoff EN, Pace NR: The human nasal microbiota and Staphylococcus aureus carriage. PLoS One 2010, 5:e10598.

7. Morris A, Beck JM, Schloss PD, Campbell TB, Crothers K, Curtis JL, Flores SC, Fontenot AP, Ghedin E, Huang L, Jablonski K, Kleerup E, Lynch SV, Sodergren E, Twigg H, Young VB, Bassis CM, Venkataraman A, Schmidt TM, Weinstock GM, Lung HIVMP: Comparison of the respiratory microbiome in healthy nonsmokers and smokers. Am J Respir Crit Care Med 2013, 187:1067-1075.

8. $\quad$ Ling Z, Liu X, Luo Y, Yuan L, Nelson KE, Wang Y, Xiang C, Li L: Pyrosequencing analysis of the human microbiota of healthy Chinese undergraduates. BMC Genomics 2013, 14:390.

9. Yong E: Gut microbial 'enterotypes' become less clear-cut. In: Nature/ News. NPG; 2012: doi:10.1038/nature.2012.10276

10. Blaser MJ, Falkow S: What are the consequences of the disappearing human microbiota? Nat Rev Microbiol 2009, 7:887-894

11. Cox MJ, Allgaier M, Taylor B, Baek MS, Huang YJ, Daly RA, Karaoz U, Andersen GL, Brown R, Fujimura KE, Wu B, Tran D, Koff J, Kleinhenz ME, Nielson D, Brodie EL, Lynch SV: Airway microbiota and pathogen abundance in age-stratified cystic fibrosis patients. PLoS One 2010, 5:e11044.

12. Huang YJ, Lynch SV, Wiener-Kronish JP: From microbe to microbiota: considering microbial community composition in infections and airway diseases. Am J Respir Crit Care Med 2012, 185:691-692.

13. Flanagan JL, Brodie EL, Weng L, Lynch SV, Garcia O, Brown R, Hugenholtz P, DeSantis TZ, Andersen GL, Wiener-Kronish JP, Bristow J: Loss of bacterial diversity during antibiotic treatment of intubated patients colonized with Pseudomonas aeruginosa. J Clin Microbio/ 2007, 45:1954-1962.

14. Huang YJ, Kim E, Cox MJ, Brodie EL, Brown R, Wiener-Kronish JP, Lynch SV: A persistent and diverse airway microbiota present during chronic obstructive pulmonary disease exacerbations. OMICS 2010, 14:9-59. 
15. Ichinohe T, Pang IK, Kumamoto Y, Peaper DR, Ho JH, Murray TS, Iwasaki A: Microbiota regulates immune defense against respiratory tract influenza A virus infection. Proc Natl Acad Sci U S A 2011, 108:5354-5359.

16. Eccles R: Understanding the symptoms of the common cold and influenza. Lancet Infect Dis 2005, 5:718-725.

17. Willner D, Furlan M, Haynes M, Schmieder R, Angly FE, Silva J, Tammadoni S, Nosrat B, Conrad D, Rohwer F: Metagenomic analysis of respiratory tract DNA viral communities in cystic fibrosis and non-cystic fibrosis individuals. PLoS One 2009, 4:e7370.

18. Hur M, Kim Y, Song HR, Kim JM, Choi YI, Yi H: Effect of genetically modified poplars on soil microbial communities during the phytoremediation of waste mine tailings. Appl Environ Microbiol 2011, 77:7611-7619.

19. Edgar RC, Haas BJ, Clemente JC, Quince C, Knight R: UCHIME improves sensitivity and speed of chimera detection. Bioinformatics 2011 27:2194-2200.

20. Kim OS, Cho YJ, Lee K, Yoon SH, Kim M, Na H, Park SC, Jeon YS, Lee JH, Yi H, Won S, Chun J: Introducing EzTaxon-e: a prokaryotic 16S rRNA gene sequence database with phylotypes that represent uncultured species. Int J Syst Evol Microbiol 2012, 62:716-721.

21. Saitou N, Nei M: The neighbor-joining method: a new method for reconstructing phylogenetic trees. Mol Biol Evol 1987, 4:406-425.

22. Kumar S, Nei M, Dudley J, Tamura K: MEGA: a biologist-centric software for evolutionary analysis of DNA and protein sequences. Brief Bioinform 2008, 9:299-306.

23. Jukes TH, Cantor CR: Evolution of protein molecules. In Mammalian Protein Metabolism. 3rd edition. Edited by Munro HN. New York: Academic Press; 1969.

24. Felsenstein J: Confidence-limits on phylogenies - an approach using the bootstrap. Evolution 1985, 39:783-791

25. Yi H, Cho YJ, Yoon SH, Park SC, Chun J: Comparative genomics of Neisseria weaveri clarifies the taxonomy of this species and identifies genetic determinants that may be associated with virulence. FEMS Microbiol Lett 2012, 328:100-105

26. Simpson EH: Measurement of diversity. Nature 1949, 163:688

27. Hamady M, Lozupone C, Knight R: Fast UniFrac: facilitating high-throughput phylogenetic analyses of microbial communities including analysis of pyrosequencing and PhyloChip data. ISME J 2010, 4:17-27.

28. Storey JD: A direct approach to false discovery rates. J Roy Stat Soc B 2002, 64:479-498.

29. Bootsma HJ, van der Heide HGJ, van de Pas S, Schouls LM, Mooi FR: Analysis of Moraxella catarrhalis by DNA typing: Evidence for a distinct subpopulation associated with virulence traits. J Infect Dis 2000, 181:1376-1387.

30. Richter M, Rossello-Mora R: Shifting the genomic gold standard for the prokaryotic species definition. Proc Natl Acad Sci U S A 2009, 106:19126-19131.

31. De Vries SPW, Bootsma HJ, Hays JP, Hermans PWM: Molecular aspects of Moraxella catarrhalis pathogenesis. Microbiol Mol Biol R 2009, 73:389-406.

32. Aas JA, Paster BJ, Stokes LN, Olsen I, Dewhirst FE: Defining the normal bacterial flora of the oral cavity. J Clin Microbiol 2005, 43:5721-5732.

33. Frandsen EV, Pedrazzoli V, Kilian M: Ecology of viridans streptococci in the oral cavity and pharynx. Oral Microbiol Immunol 1991, 6:129-133.

34. Arumugam M, Raes J, Pelletier E, Le Paslier D, Yamada T, Mende DR, Fernandes GR, Tap J, Bruls T, Batto JM, Bertalan M, Borruel N, Casellas F, Fernandez L, Gautier L, Hansen T, Hattori M, Hayashi T, Kleerebezem M, Kurokawa K, Leclerc M, Levenez F, Manichanh C, Nielsen HB, Nielsen T, Pons N, Poulain J, Qin J, Sicheritz-Ponten T, Tims S, et al: Enterotypes of the human gut microbiome. Nature 2011, 473:174-180.

35. Koren O, Knights D, Gonzalez A, Waldron L, Segata N, Knight R, Huttenhower C, Ley RE: A guide to enterotypes across the human body: meta-analysis of microbial community structures in human microbiome datasets. PLoS Comput Biol 2013, 9:e1002863.

36. Bottone $\mathrm{E}$, Allerhan J: Association of mucoid encapsulated Moraxella duplex var. nonliquefaciens with chronic bronchitis. Appl Microbiol 1968, 16:315-319.

37. Henriksen SD: Moraxella duplex var. nonliquefaciens as a cause of bronchial infection. Acta Pathol Mic Sc 1951, 29:258-262.

38. Rosett W, Heck DM, Hodges GR: Pneumonitis and pulmonary abscess associated with Moraxella nonliquefaciens. Chest 1976, 70:664-665.

39. Laukeland $\mathrm{H}$, Bergh $\mathrm{K}$, Bevanger L: Posttrabeculectomy endophthalmitis caused by Moraxella nonliquefaciens. J Clin Microbiol 2002, 40:2668-2670.
40. Johnson DW, Lum G, Nimmo G, Hawley CM: Moraxella nonliquefaciens septic arthritis in a patient undergoing hemodialysis. Clin Infect Dis 1995, 21:1039-1040

41. Sudar JM, Alleman MJA, Jonkers GJPM, Degroot R, Jongejan C: Acute thyroiditis caused by Moraxella nonliquefaciens. Neth J Med 1994, 45:170-173.

42. Lefebvre S, Vanbosterhaut B, Wauters G: Discitis due to Moraxella nonliquefaciens. Rev Rhum 1994, 61:501-501.

43. Feldman SR, Petersen MJ: Botryomycosis caused by Moraxella nonliquefaciens. Cutis 1989, 43:140-142.

44. Bechard DL, Lefrock JL, Tillotson JR: Endocarditis caused by Moraxella nonliquefaciens. Southern Med J 1979, 72:1485-1487.

45. Bøvre K: Genus II. Moraxella Lwoff 1939, 173 emend. Henriksen and Bøvre 1968, 391 ${ }^{\mathrm{AL}}$. In Bergey's Manual of Systematic Bacteriology. 1st edition. Edited by Krieg NR, Holt G. Baltimore: Williams \& Wilkins; 1984:296-303.

46. Tønjum T, Marrs CF, Rozsa F, Bøvre K: The type-4 pilin of Moraxella nonliquefaciens exhibits unique similarities with the pilins of Neisseria gonorrhoeae and Dichelobacter (Bacteroides) nodosus. J Gen Microbio 1991, 137:2483-2490.

47. Murphy TF, Parameswaran Gl: Moraxella catarrhalis, a human respiratory tract pathogen. Clin Infect Dis 2009, 49:124-131.

48. Verhaegh SJ, Streefland A, Dewnarain JK, Farrell DJ, Van Belkum A, Hays JP. Age-related genotypic and phenotypic differences in Moraxella catarrhalis isolates from children and adults presenting with respiratory disease in 2001-2002. Microbiology 2008, 154:1178-1184.

49. Verduin $\mathrm{CM}, \mathrm{Hol}$ C, Fleer A, Van Dijk H, Van Belkum A: Moraxella catarrhalis: from emerging to established pathogen. Clin Microbiol Rev 2002, 15:125-144.

50. Faden $\mathrm{H}$, Harabuchi $\mathrm{Y}$, Hong JJ: Epidemiology of Moraxella catarrhalis in children during the first 2 years of life: relationship to otitis media. J Infect Dis 1994, 169:1312-1317.

51. Faden $\mathrm{H}$ : Comparison of the local immune response to nontypable Haemophilus influenzae $(\mathrm{nHI})$ and Moraxella catarrhalis (MC) during otitis media. Adv Exp Med Biol 1995, 371B:733-736.

52. Van Hare GF, Shurin PA, Marchant CD, Cartelli NA, Johnson CE, Fulton D, Carlin S, Kim CH: Acute otitis media caused by Branhamella catarrhalis: biology and therapy. Rev Infect Dis 1987, 9:16-27.

53. Varon E, Levy C, De La Rocque F, Boucherat M, Deforche D, Podglajen I, Navel M, Cohen R: Impact of antimicrobial therapy on nasopharyngeal carriage of Streptococcus pneumoniae, Haemophilus influenzae, and Branhamella catarrhalis in children with respiratory tract infections. Clin Infect Dis 2000, 31:477-481.

54. Digiovanni C, Riley TV, Hoyne GF, Yeo R, Cooksey P: Respiratory-tract infections due to Branhamella catarrhalis - epidemiologic data from Western-Australia. Epidemiol Infect 1987, 99:445-453.

55. Bootsma HJ, Van Dijk H, Vauterin P, Verhoef J, Mooi FR: Genesis of BRO beta-lactamase-producing Moraxella catarrhalis: evidence for transformation-mediated horizontal transfer. Mol Microbiol 2000 36:93-104

56. De Vries SP, Van Hijum SA, Schueler W, Riesbeck K, Hays JP, Hermans PW, Bootsma HJ: Genome analysis of Moraxella catarrhalis strain BBH18, a human respiratory tract pathogen. J Bacterio/ 2010, 192:3574-3583.

doi:10.1186/s12879-014-0583-3

Cite this article as: Yi et al.: Profiling bacterial community in upper respiratory tracts. BMC Infectious Diseases 2014 14:583.

\section{Submit your next manuscript to BioMed Central and take full advantage of:}

- Convenient online submission

- Thorough peer review

- No space constraints or color figure charges

- Immediate publication on acceptance

- Inclusion in PubMed, CAS, Scopus and Google Scholar

- Research which is freely available for redistribution 